Z. klin. Chem. u. klin. Biochem.

9. Jg., S. 18-20, Januar 1971

\title{
Eine quantitative Bestimmungsmethode von, 3-Methoxy-4-hydroxy-mandelsäure (Vanillinmandelsäure) aus dem Urin
}

\author{
Von J. Gartzke und E. Majewski \\ Aus dem Medizinisch-Diagnostischen Institut „Unter den Linden", Berlin, Leiter: Dr. K. H. Goll
}

(Eingegangen am 7. Juli 1970)

Es wird eine kolorimetrische Bestimmungsmethode der Vanillinmandelsäure im Urin nach dünnschichtchiromatographischer Abtrennung an Kieselgel G mit sauren Laufmitteln durch Reaktion mit 2.6-Dichlor-4-chlor-chinonimid in Gegenwart von Pyridin als Katalysator beschrieben. Die obere Grenze des Normalwertes liegt nach unseren Erfahrungen bei $5 \mathrm{mg} / 24$ Stdn.-Harn.

\section{A quantitative method for the determination of 3-methoxy-4-bydroxy-mandelic acid (vanillinmandelic acid) in urine}

A colorimetric method is described for the determination of urinary vanillinmandelic acid. After separation by thin layer chromatography on Kieselgel $\mathrm{G}$ with acidic solvents, the colour is developed by reacting the vanillinmandelic acid with 2.6-dichlor-4-chlor-quinonimide in the presence of pyridine as a catalyst. In the present work, the upper limit for the normal value was found to be $5 \mathrm{mg} / 24 \mathrm{hr} u r i n e$.

Zur Diagnose von Phäochromocytomen gewinnt die Bestimmung der Adrenalin- und Noradrenalinmetabolite im Harn ständig an Bedeutung. Durch Tumoren an den katecholaminausscheidenden Organen wird die Sekretion von Nor- und Adrenalin erhöht, so daß es zu Blutdruckkrisen oder einer permanenten Hypertonie kommt. Es werden aber nur etwa 10\% der endogenen Katecholamine unverändert im Urin ausgeschieden, $40-60 \%$ in Form des Metaboliten 3-Methoxy-4-hydroxy-mandelsäure (Vanillinmandelsäure) (1).

Bei den bisherigen Bestimmungsmethoden wird die Vanillinmandelsäure elektrophoretisch $(2,3,4)$, papier$(1,5)$, säulen- $(6,7)$, gas- $(8,9)$ oder dünnschichtchromatographisch $(10,11,12,13)$ von den anderen Phenolcarbonsäuren abgetrennt. Die quantitative Bestimmung erfolgt entweder ultraviolett-spektrophotometrisch $(14,15)$ oder densitometrisch $(16,17)$ bzw. kolorimetrisch nach Kupplung mit Diazoniumsalzen $(10,11)$, Oxydation der Vanillinmandelsäure zu Vanillin und Reaktion mit Indol (7), Reaktion mit GIBBS Reagenz (2.6-Dichlor- oder 2.6-Dibrom-chinon-4-chlorimid) $(17,18)$.

Bei der von uns dünnschichtchromatographisch angestrebten Methode entschlossen wir uns nach Vorversuchen für Kieselgel G (Merck) unter Verwendung saurer Laufmittel, die eine optimale Trennung gewährleisten. Basische Laufmittel (10) sind zur Trennung weniger geeignet. Nachteilig bei der Verwendung von Kieselgel $G$ ist die Einschränkung der Anfärbemethoden. Während die Kupplung mit diazotiertem $p$-Nitranilin auf Celluloseplatten stark rotviolett gefärbte Vanillinmandelsäure-Banden mit guter Farbkonstanz liefert, erhält man bei gleicher Reaktion auf Kieselgel $G^{i}$ nur rötlich bis braune Vanillinmandelsäure-Zonen. McGREGOR (16) versuchte zur densitometrischen Auswertung diesen Mangel zu beheben, indem er die angefärbten Vanillinmandelsäure-Banden mit Dimethylsulfoxid nachbehandelte. Doch ist auch hierbei die Farbkonstanz nach unseren Erfahrungen für densitometrische Auswertung noch nicht ausreichend.

Theoretische Betrachtungen zeigten, daß Gibss Reagenz das Mittel der Wahl ist, wenn ein entscheidender Nachteil - der der nicht vollständigen Umsetzung behoben wird. Von den 16 bis 24 theoretisch im Harn vorkommenden Phenolcarbonsäuren können nur noch 11 Säuren eine GrbBs-Reaktion eingehen. Sterische Hinderungen und elektromere Effekte lassen von den 11 restlichen Säuren noch 4 bis 6 eine GrbBs-Reaktion eingehen. Ein weiterer Vorteil ist, daß sich das Absorptionsspektrum der angefärbten Vanillinmandelsäure nicht mit denen der mitextrahierten Harnchromogene überlagert. Ferner wurde versucht, eine möglichst selektive Extraktion der Vanillinmandelsäure zu erreichen. Dazu ist entgegen anderen Angaben $(2,19)$ Äthylacetat das einzig brauchbare Extraktionsmittel.

\section{Versuche zur Methode}

Extraktion

Die von manchen Autoren (2) - der Extraktion mit Essigester gleichwertig hingestellte Extraktion mit Äther liefert, wie der Vergleich der Verteilungskoeffizienten beider Systeme zeigt, nur schlechte Ausbeuten. Erst eine 6malige Extraktion von 1 Teil. Harn mit jeweils 5,5 Teilen Ather lieferte eine maximale Ausbeute. Wir extrahieren $4 \mathrm{mal}$ mit jeweils 1,6 Teilen Essigester pro 1 Teil Harn. Uberprüft wurden die Ausbeuten durch kontinuierliche Extraktion des Harns mit A ther in einem KUTSCHER-STEUDELPerforator.

Dünnschichtchromatographie und Elution

Als stationäre Phase verwendeten wir Kieselgel G (Merck). Zur besseren Trennung wendeten wir die Keilstreifentechnik in Kombination mit einer Zweifachentwicklung an, doch war die Trennung mit dem ammoniakalischen Laufmittel von STROBACH (10) unbefriedigend. Wir fanden in Anlehnung an dieses Laufmittel bei Verwendung von Puffern anstelle von Ammoniak bei $\mathrm{pH}$ 3,8 die besten Trennungen. Wir versuchten daher das von Pastusía (20) angegebene Laufmittel Eisessig: Benzol: Methanol $=4: 45: 8(\mathrm{v} / \mathrm{v})$, das dann aúch die besten Trennungen 
zeigtc. Dic Elution erfolgt quantitativ. Ein eventucller Verlust tritt, wic sich aus Abbildung 1 deuten läßt, möglicherweise durch die eigentliche Chromatographie ein.

Anfärbung der Vanillinmandelsäure

Den Vorteilen der Anfärbung mit Grbss Reagenz, bessere Trennung der Flecke voneinander, da sich weniger Phenolcarbonsäuren anfärben als mit diazotiertem p-Nitranilin, keine Uberlappung der Spektren der angefärbten Vanillinmandelsäure mit denen der Harnchromogene, stehen die Nachteile der pH-Abhängigkeit der Reaktionsgeschwindigkeit, der Farbintensität- und -konstanz gegenüber. Alle Nachteile werden bei Verwendung von Boratpuffer pH 9,5 mit 10\% Pyridin als Katalysator behoben. Diese Pyridin-Konzentration hat sich als optimal erwiesen. Dic kolorimetrische Messung erfolgt nach 25 Minuten bei $620 \mathrm{~nm}$ im Absorptionsmaximum der reinen mit GiBss Reagenz angefärbten Vanillinmandelsäure. Das Absorptionsmaximum der Harn-Vanillinsäure ist um etwa $10 \mathrm{~nm}$ in den kürzerwelligen Bercich verschoben. Abbildung 2 zeigt die Absorptionsspektren der 4 wichtigsten im Harnextrakt mit GibBs Reagenz anfärbbaren Phenolcarbonsäuren (Abb. 3). Die zugesetzte Menge 2.6-Dichlorchinon-4-chlorimid ist ausreichend für mindestens $150 \mu \mathrm{g} \mathrm{Va}$ nillinmandelsäure pro Fleck auf dem Dünnschichtchromatogramm, doch ist die Extinktion nur bis etwa $50 \mu \mathrm{g}$ Vanillinmandelsäure streng linear.

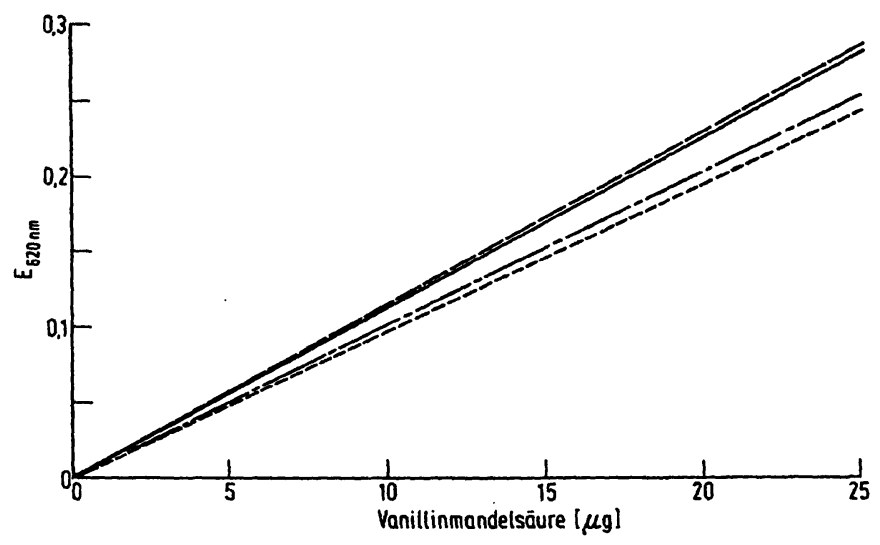

Abb. 1

Eichkurven:

reine Vanillinmandelsäure

reine Vanillinmandelsäure nach Elution von Kieselgel $G$ ohne Dünnschichtchromatographie (DC)

reine Vanillinmandelsäure nach $D C$ und Elution

Vanillinmandelsäure plus Harnextrakt nach DC und Elution gemessen gegen Harnextrakt (Spekol, $620 \mathrm{~nm}$ )

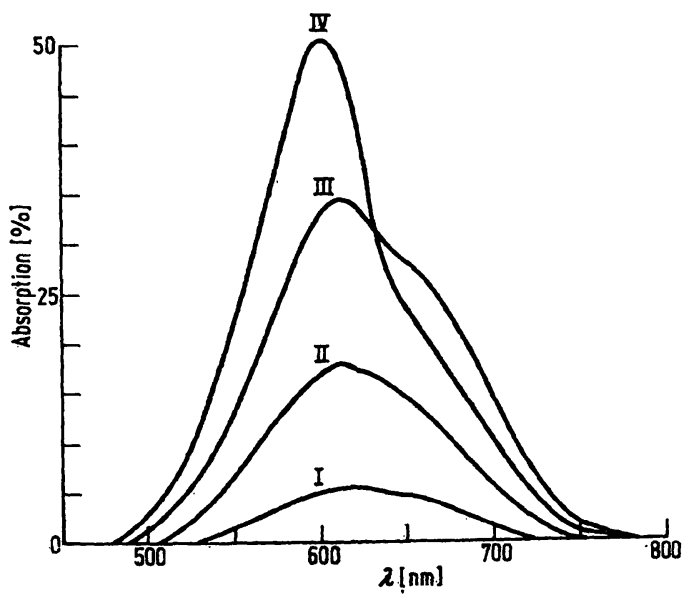

Abb. 2 Absorptionsspektren der vier in Abb. 3 mit GiBss Reagenz angefärb-
ten Phenolcarbonsäuren (II $=$ Vanillinmandelsäure; 1, III, IV $=$ nicht identifiziert, Beckman - Spektrophotometer DU;

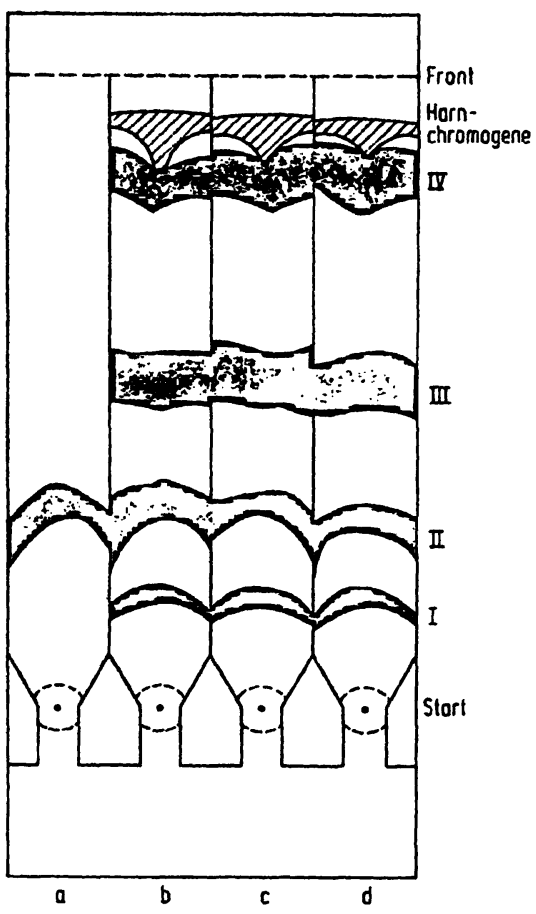

Abb. 3

Verteilung der einzelnen Fraktionen auf dem Chromatogramm: I, III IV nicht identifizierte Phenolcarbonsäuren, II $=\mathrm{a}$ : Vanillinnaandelsäure-Standard; $b$ : Vanillinmandelsäure-Standard plus Harnextrakt;
c, d: Harnextrakt

\section{Methodik}

Prinzip

Anteile des zu untersuchenden 24-Stdn.-Sammelharns werden mit Salzsäure angesäuert ( $\mathrm{pH} 1), \mathrm{NaCl}$ gesättigt und Essigester extrahiert. Der über Natriumsulfat getrocknete Essigesterextrakt wird im Rotationsverdampfer zur Trockne eingedampft, der Rückstand in 60 proz. Alkohol aufgenommen. Aufnehmen in wäßr. Methanol ist zu vermeiden (21). Nach dünnschichtchromatographischer Abtrennung der Vanillinmandelsäure an Kieselgel $G$ wird mit GrbBs Reagenz besprüht, die VanillinmandelsäureBande abgekratzt und mit Boratpuffer/Grass-Reagenz/Pyridin eluiert, kolorimetrisch bestimmt und mit einem auf gleicher Platte mitgelaufenen Standard berechnet.

\section{Reagenzien}

1. konz. Salzsäure

2. Natriumchlorid p. a.

3. Äthylacetat p. a.

4. Natriumsulfat sicc. DAB 7

5. 60 proz. Äthanol

6. Kieselgel G (Merck)

7. Benzol (zur Molekulargewichtsbestimmung)

8. Methanol p. a.

9. Eisessig p. a.

10. Borat-Citrat-Puffer $\mathrm{pH} 9,5$

$3,54 \mathrm{~g}$ Borsäure und $7,0 \mathrm{~g}$ Citronensäure werden in $900 \mathrm{ml}$ Wasser gelöst und unter Rühren $13,7 \mathrm{~g}$ festes Natriumhydroxid zugefügt. Nach Lösen werden $2,23 \mathrm{ml} 85$ proz. Phosphorsäure zugegeben und unter Kontrolle des pHWertes mit einem pH-Meter mit konz. Salzsäure auf $\mathrm{pH}$ 9,5 gebracht.

11. Pyridin reinst (frisch destillicrt)

12. 2.6-Dichlor-chinon-4-chlorimid reinst

13. Sprühreagenz $(0,1$ proz. Gibss-Reagenz in Alkohol, Aufbewahrung im Kühlschrank)

14. Vanillinmandelsäure-Standard ( $1 \mathrm{mg} / \mathrm{m} l$ Vanillinmandelsäure p. a.). 


\section{Vorschrift}

$50 \mathrm{~m} / 24-\mathrm{Stdn}$.-Sammelharn (es kann auch entsprechend weniger genommen werden) werden mit konz. $\mathrm{HCl}$ auf pH 1 gebracht und mit Natriumchlorid gesättigt. Anschließend wird der Harn $4 \mathrm{mal}$ mit je $30 \mathrm{ml}$ Essigester extrahiert (zur Phasentrennung bei Emulsionsbildung zentrifugieren), die vereinigten Essigesterextrakte werden über Natriumsulfat getrocknet. Der getrocknete Essigester wird im Vakuum zur Trockne gebracht und der Rïckstand in $0,4 \mathrm{ml} 60$ proz. Äthanol gelöst.

Die Dünnschichtchromatographie erfolgt auf Keilstreifen an Kieselgel $G$ (Schichtdicke 0,25 mm) als stationäre und Eisessig-Benzol-Methanol $(4+45+8$ $v / v)$ als mobile Phase. Aufgetragen werden ein Vanilinmandelsäure-Standard (10 $\mu \mathrm{g}$ Vanillinmandelsäure), $2 \mathrm{mal} 0,03 \mathrm{~m} l$ der zu untersuchenden Probe und $1 \mathrm{mal}$ $0,03 \mathrm{ml}$ der Probe plus Vanillinmandelsäure-Standard (als Leitchromatogramm und zur Überprüfung der gefundenen Extinktionen). Zur besseren Trennung kann eine eindimensionlae Zweifachentwicklung vorgenommen werden. Die fertigentwickelte Platte wird zur Abstumpfung der Essigsäure 2-3 Min. in eine $\mathrm{NH}_{3}$-Kammer gestellt, dann mit Boratpuffer $\mathrm{pH} 9,5$ besprüht und getrocknet. Nach Besprühen mit GisBS Sprühreagenz wird die Platte zur Farbvertiefung einige Sekunden einer $\mathrm{NH}_{3}$-Atmosphäre ausgesetzt, die Vanillinmandelsäure-Bande abgekratzt und mit $5,4 \mathrm{ml}$ Boratpuffer $\mathrm{pH} 9,5,0,6 \mathrm{~m} l$ Pyridin und $0,1 \mathrm{~m} l$ Sprühreagenz eluiert. Nach $15 \mathrm{Min}$. langem Stehen wird $10 \mathrm{Min}$. bei 3500 U/Min. zentrifugiert, die überstehende Lösung abdekantiert, bei $620 \mathrm{~nm}$ und einer Schichtdicke $\mathrm{d}=1 \mathrm{~cm}$ gegen einen Blindwert gemessen, der der Leitbahn des Standardwertes entnommen wurde. Es wird wegen der möglichen Fehlerquellen beim Besprühen der DC-Platten unbedingt die Berechnung mit einem Standard empfohlen, da die Extinktionen des $10 \mu \mathrm{g}$ Vanillinmandelsäure-Standards auf den Chromatogrammen maximal $\pm 20 \%$ schwanken können. Beim Vergleich der gefundenen Werte der Vanillinmandelsäure-Ausscheidung nach Anfärbung mit diazotiertem $p$-Nitranilin und Gibis's Reagenz waren die Differenzen $z$. T. erheblich, es wurden nach Anfärbung mit Grbss Reagenz meist weniger, und zwar durchschnittlich $25 \%$ weniger Vanillinmandelsäure gefunden als nach Anfärbung mit diazotiertem $p$-Nitranilin. Dies deutet auf eine selektivere Bestimmung der Vanillinmandelsäure mit GrbBs Reagenz hin.

Die von Strobach (10) und anderen Autoren angegebenen Normalwerte erscheinen uns aufgrund theoretischer Überlegungen der Katecholaminausscheidung sowie deren Metabolisierungstaten zu hoch. Sie dürften maximal bei $5 \mathrm{mg} / 24$-Stdn.-Harn $\pm 10 \%$ mit einem Grenzbereich bis $6 \mathrm{mg}$ liegen. Dies würde unseren, anhand von 33 verschiedenen Harnen, deren Werte im Normalbereich lagen, errechneten Mittelwert von 3,45 mg/24-Stdn.-Harn mit einer Standardabweichung von $s= \pm 1,27$ sowie den von Preston (11) angegebenen Werten etwa entsprechen. Als Maß der Streuung wird allgemein $\pm 2,3 \mathrm{~s}$ angegeben, wonach unser Mittelwert den Betrag von 3,45 $\pm 2,92 \mathrm{mg} / 24-\mathrm{Stdn}$.Harn annimmt. In dieser Streuung ist sowohl die methodische als auch biologische enthalten. Bei Vergleichen beider Methoden legten wir für Anfärbungen mit diazotiertem $p$-Nitranilin Normalwețte von $7 \mathrm{mg}$ (10) und für Anfärbungen mit GrbBs Reagenz $5 \mathrm{mg} /$ 24-Stdn.-Harn zugrunde. Von 8 untersuchten pathologischen Patientenharnen waren 5 Harne nach beiden Anfärbungen pathologisch, 2 Harne nur nach Anfärbung mit diazotiertem $p$-Nitranilin und 1 Harn nur nach Anfärbung mit GrBBs Reagenz.

\section{Literatur}

1. Gitlow, S. E., M. Mendlowrtz, S. Khassis, G. Cohen und J. Sha, J. Clin., Invest. 39, 221 (1960). - 2. Studnitz, W. v., Scand. J. Clin. Laborat. Invest. 12 Suppl. No. 48, 1-73 (1960). 3. Butrer, T. J., Clin. Chem. New York, 13, 488 (1967). - 4. O'Goraran, L. P., Clin. Chem. Acta Amsterdam, 19, 485 (1968). 5. Duke, P. S. und H. B. Demopoulos, Clin. Chim. New York, 14, 212 (1968). - 6. Bajor, G. F. und W. G. Clark, J. Chromatog. 14, 447 (1964). - 7. Wybenga, D. und V. J. Pileggr, Clin. Chim. Acta Amsterdam, 16, 147 (1967). - 8. KARONIN, F., C. R. J. Ruthrien und M. SANdler, Clin. Chim. Acta Amsterdam, 20, 427 (1968).'-' 9.'GREer, M., [T.'JJ. SpRINKLE und C. M. WilLIAMS, Clin. Chim. Acta Amsterdam, 21, 247 (1968). - 10. Strobach, H., diese Z. 5,77 (1967). - 11. Presten, J. A., Clin. Chem. New
York, 13, 19 (1967). - 12. Dittmann, J. und G. Liem, diese Z 4, 265 (1968). - 13. Segura-Cardona, R. und K. Soemring, Med. exp. 10, 251 (1964). - 14. De Potter, W. P., R. F. Vochten und A. F. DE SchaEpdryver, Experientia Basel, 21, 482 (1965). 15. Nakagawa, M. R. Shetrar und S. H. Wender, Analytic. Biochem. 7, 374 (1964). - 16. McGregor, R. F., M. KHAN, D. Marrack und M. P. Suvillan, Amet. J. Clin. Pathol. 46, 163 (1965). - 17. Köhler, P. und H. Baufeld, Ärztl. Lab. 10, 224 (1964). - 18. SmIrt, P., West European Symp. Clin. Chem. 2, 31, 97 (1963). - 19. Fellman, J. H., L. J. Severson, E. H. Robinson undT. S. Fujtra, Amer. J. Clin. Pathol., 38, 651 (1962). - 20. Pastuska, G., Z. analyt. Chem. 179, 355 (1961). 21. DirscherL, W., diese Z. 7, 200 (1969).

Dipl.-Chem. J. Gartzke u. E. Majewski $\mathrm{X} 108$ Berlin

Unter den Linden 40 


\section{Sephadex

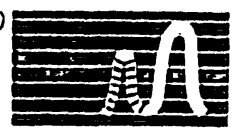

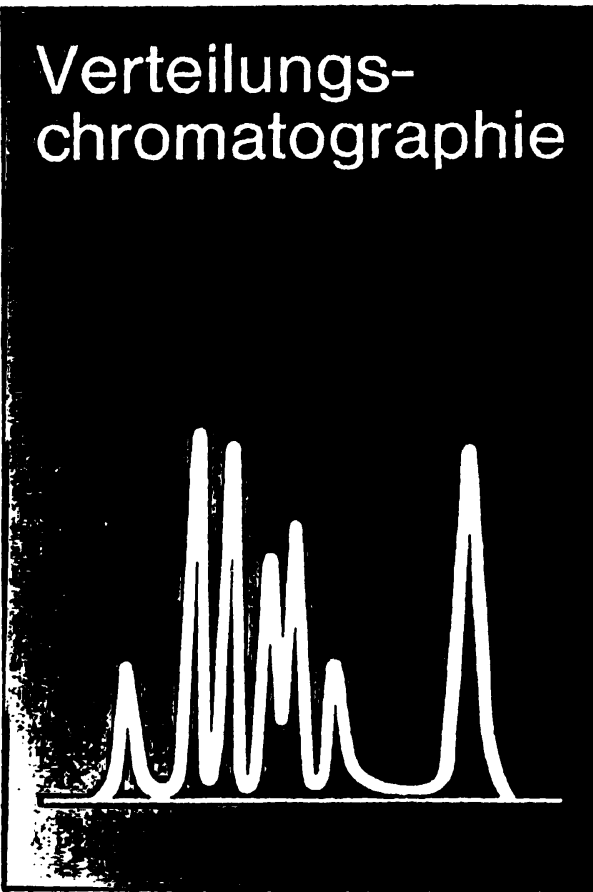

Trennung voresterter Catecholamin-Metaboliten durch Verteilungschromatographio in 1,2-Dichlorathan/Mothanol 7:3.

(E. ÁNGGARD ot al., J. Chromatog. 50 (1970) 251-259.)

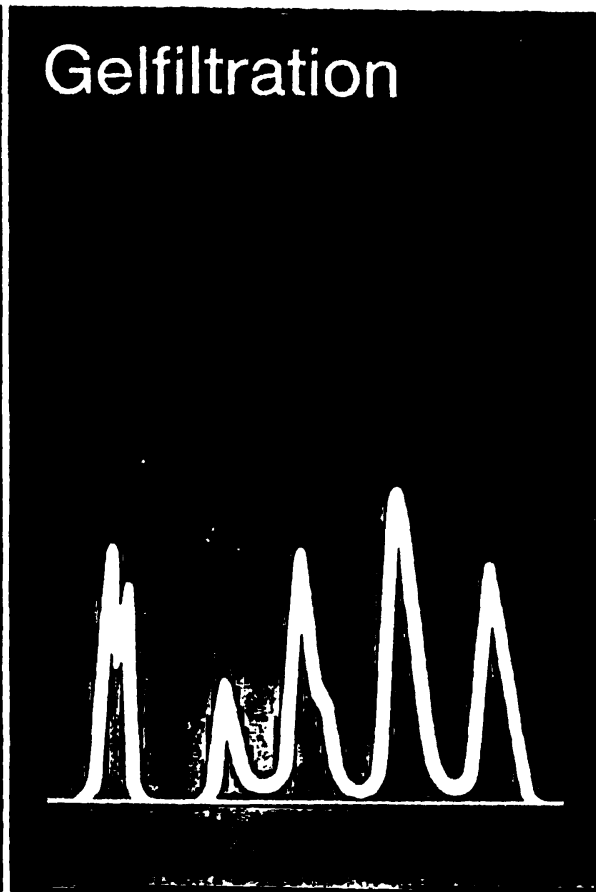

Gelfiltration von Triglycoridon und Fellsäuren in Chloroform.

(W. K. DOWNEY of al., J. Chromatog. 46 (1970) 120-124.)

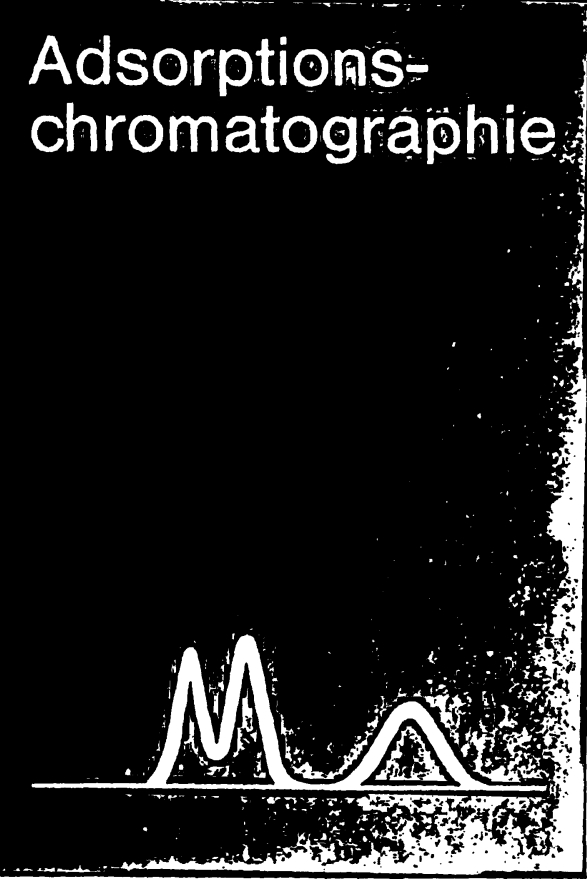

Trennung von Ostrogenon durch Adsorptionschromalographio in dest. Wassor. (H. van BAELEN ot al., J. Chromatog. 30 (1867) 226-227.)

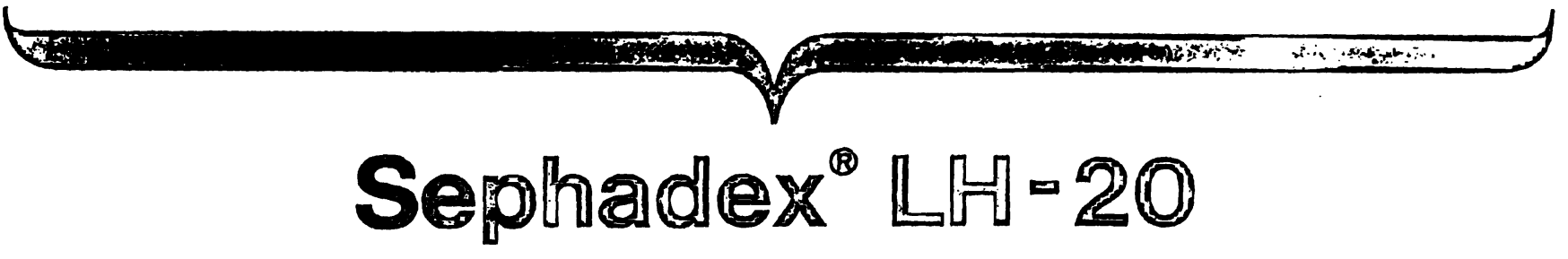

\section{trennt in organischen Lösungsmitteln und in wäßrigen Lösungen}

Sephadex LH-20 ist ein lipophiles Sephadex-Derivat und wird durch Hydroxypropylieren aus Sephadex G-25 gewonnen. Es wird in Perlform (Partikelgröße trokken 25-100 $\mu$ ) als freifließendes Pulver geliefert, das in Wasser und polaren organischen Lösungsmitteln quillt. Der Quellgrad nimmt mit abnehmender Polarität der Lösungsmittel ab. Sephadex LH-2O ist als Molekularsieb für die Gelfiltration in organischen Lösungsmitteln entwickelt worden, es hat sich jedoch auch für andere chromatographische
Verfahren rasch durchgesetzt, die auf Verteilungs- und Adsorptionseffekten beruhen.

Sephadex LH-20 wird eingesetzt in der Fraktionierung von Polymeren, zur Trennung von Lipiden und Steroiden, zum Separieren von aromatischen und aliphatischen Kohlenwasserstoffen sowie zur Lösung vieler anderer Trennprobleme.

Für das Arbeiten in Lösungsmittelsystemen haben wir spezielle lösungsmittelfeste Chromatographierohre entwickelt. 

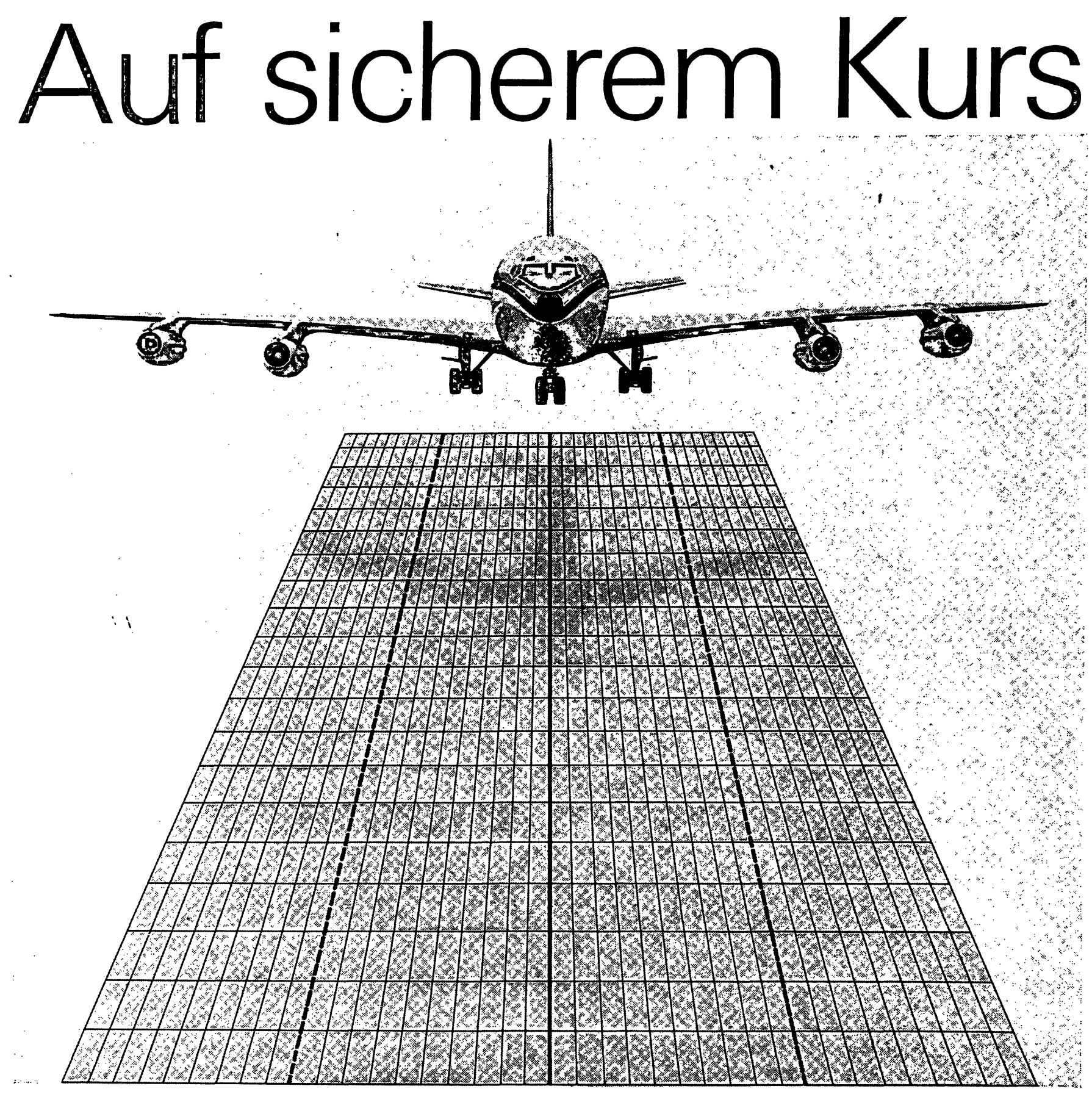

\section{Qualitätskontrolle im klinisch-chemischen Labor mit}
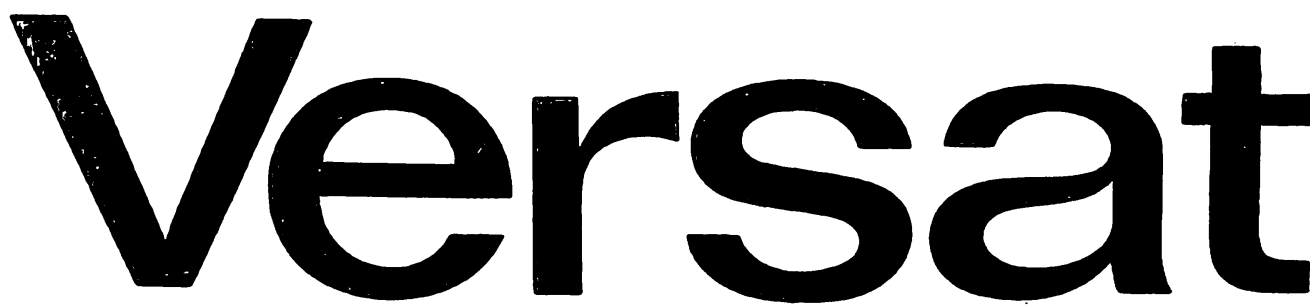

(R)

\section{Standard- und Kontrollseren}

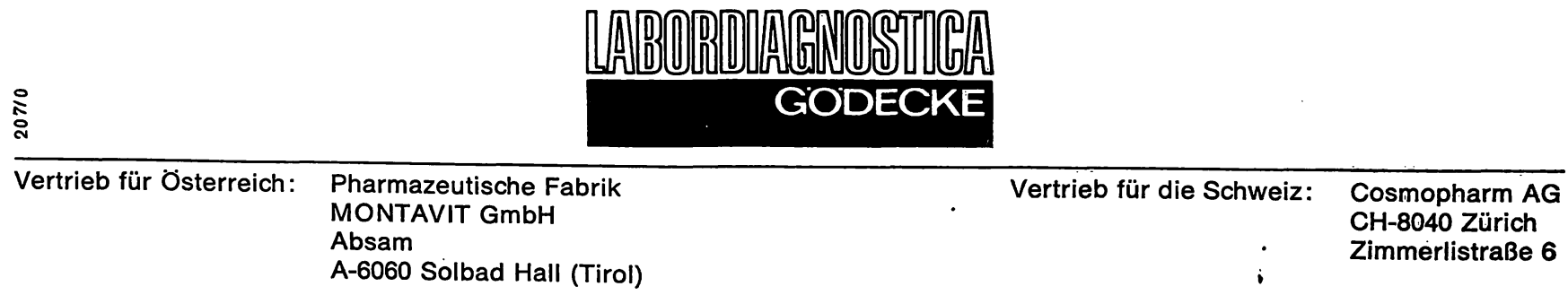

